\title{
On the stability of AdS black strings
}

\author{
Yves Brihaye, ${ }^{\dagger}$ Terence Delsate ${ }^{\dagger}$ and Eugen Radu ${ }^{\ddagger}$ \\ ${ }^{\dagger}$ Physique-Mathématique, Universite de Mons-Hainaut, Mons, Belgium \\ ${ }^{\ddagger}$ Laboratoire de Mathématiques et Physique Théorique, Université François-Rabelais, Tours, France
}

October 27, 2018

\begin{abstract}
We explore via linearized perturbation theory the Gregory-Laflamme instability of the black string solutions of Einstein's equations with negative cosmological constant recently discussed in literature. Our results indicate that the black strings whose conformal infinity is the product of time and $S^{d-3} \times S^{1}$ are stable for large enough values of the event horizon radius. All topological black strings are also classically stable. We argue that this provides an explicit realization of the Gubser-Mitra conjecture.
\end{abstract}

\section{Introduction}

In 1993, Gregory and Laflamme (GL) made the surprising discovery that the extended black objects appearing in higher dimensions are classically unstable [1. The simplest extended black object in general relativity is found by trivially extending to $d$-spacetime dimensions the Schwarzschild black hole solution in $d-1$ dimensions and corresponds to a uniform black string (UBS) with horizon topology $S^{d-3} \times S^{1}$. The results in [1] showed that this configuration is classically unstable below a critical value of the mass. Following this discovery, a branch of nonuniform black string (NUBS) solutions was found perturbatively from the critical GL string [2, 3, 4. This nonuniform branch was subsequently numerically extended into the full nonlinear regime in [3], [5], 6] (see [7, [8 for reviews of this topic).

Most of the work on the stability and phases of black strings have been performed so far assuming a vanishing cosmological constant. However, recently there has been some interest in finding black string solutions with a cosmological constant $\Lambda$. The anti-de Sitter (AdS) natural counterparts of the Schwarzschild black string have been considered for the first time in [9, for the $d=5$ case. These UBS solutions are very different from the warped AdS black strings as discussed for instance in [10] (see also [17]).

For example, they have no dependence on the 'compact' extra dimension, and their conformal boundary is the product of time and $S^{2} \times S^{1}$. Higher dimensional $d>5$ black strings have been constructed in [11, configurations with an event horizon topology $H^{d-3} \times S^{1}$ being considered as well. As argued in 19, 11, these solutions provide the gravity dual of a field theory on a $S^{d-3} \times S^{1} \times S^{1}$ (or $H^{d-3} \times S^{1} \times S^{1}$ ) background. Various other examples of AdS black strings, including solutions with matter fields, can be found in [12, [13, [14. Black string with a positive cosmological constant have been constructed in [15]. Different from the $\Lambda=0$ limit, the AdS black string solutions with an event horizon topology $S^{d-3} \times S^{1}$ have a nontrivial, globally regular limit with zero event horizon radius [11.

However, the issue of the classical stability of the AdS black strings has not yet been addressed in the literature. On general grounds, one expects a richer structure in this case, since the cosmological constant introduces another scale in the theory. This type of solutions also provides a new laboratory to test the Gubser-Mitra (GM) conjecture [16], that correlates the dynamical and thermodynamical stability for systems with translational symmetry and infinite extent. In this conjecture, the appearance of a negative specific heat is related to the onset of a classical instability. This conjecture has passed a large number of tests [8], 17, but it is also known to fail in certain cases [18. Since thermodynamical stability of the AdS black strings is possible for $\Lambda<0$ [11], one expects that some of these objects do not present the GL instability. 
The paper is structured as follows: we begin with a review of the general properties of the AdS UBS solutions. In Section 3, we consider the issue of GL instability. Expanding around the UBS and solving the eigenvalue problem numerically, our results indicate that the GL instability persists up to a critical configuration only. The final Section contains a discussion of the GM conjecture for AdS black strings together with our conclusions.

\section{AdS uniform black strings}

These solutions of the Einstein equations with a negative cosmological constant $\Lambda=-(d-1)(d-2) /\left(2 \ell^{2}\right)$ have been found for a metric ansatz with three unknown functions [11]

$$
d s^{2}=-b(r) d t^{2}+\left(\frac{d r^{2}}{f(r)}+a(r) d z^{2}\right)+r^{2} d \Sigma_{\kappa}^{2},
$$

where the $(d-3)$-dimensional metric $d \Sigma_{\kappa, d-3}^{2}$ is

$$
d \Sigma_{\kappa, d-3}^{2}= \begin{cases}d \Omega_{d-3}^{2} & \text { for } \kappa=+1 \\ \sum_{i=1}^{d-3} d x_{i}^{2} & \text { for } \kappa=0 \\ d \Xi_{d-3}^{2} & \text { for } \kappa=-1,\end{cases}
$$

with $d \Omega_{d-3}^{2}$ the unit metric on $S^{d-3}$; by $H^{d-3}$ we will understand the $(d-3)$-dimensional hyperbolic space, whose unit metric $d \Xi_{d-3}^{2}$ can be obtained by analytic continuation of that on $S^{d-3}$. As usual with black strings, the coordinate along the compact direction is denoted by $z$ and its asymptotic length is $L$.

Unfortunately, for $\Lambda \neq 0$, the equations satisfied by the metric functions $a, b$ and $f$ can be solved only numerically (these equations are presented in 11]). However, one can write an approximate form of the solutions valid near the event horizon or for large values of the radial coordinate $r$. The event horizon is taken at constant $r=r_{h}$, where one finds an expansion in terms of two parameters $a_{h}, b_{1}$

$$
a(r)=a_{h}+O\left(r-r_{h}\right), b(r)=b_{1}\left(r-r_{h}\right)+O\left(r-r_{h}\right)^{2}, f(r)=\bar{f}_{1}\left(r-r_{h}\right)+O\left(r-r_{h}\right)^{2},
$$

with $\bar{f}_{1}=\left((d-1) r_{h}^{2}+k(d-4) \ell^{2}\right) /\left(r_{h} \ell^{2}\right)$. The condition for a regular event horizon is $\bar{f}_{1}>0, b_{1}>0$. In the $\kappa=-1$ case, this implies the existence of a minimal value of $r_{h}$. However, for any value of $\kappa$, there is no upper bound on the event horizon radius. In Refs. 9, 11 arguments for the existence of a nontrivial globally regular solutions with $r_{h}=0$ in the case $\kappa=1$ are presented. Also, for $\kappa=0$, the Einstein equations admit the exact solution $a=r^{2}, f=1 / b=-2 M / r^{d-3}+r^{2} / \ell^{2}$, which appears to be unique, corresponding to the known planar topological black hole with a periodic $z$-direction.

The expression of the solution near the boundary at infinity depends on two constants $c_{t}$ and $c_{z}$, which fix the UBS mass and tension. For even $d$, the solution admits at large $r$ a power series expansion of the form:

$$
\begin{gathered}
a(r)=\frac{r^{2}}{\ell^{2}}+\sum_{j=0}^{(d-4) / 2} a_{j}\left(\frac{\ell}{r}\right)^{2 j}+c_{z}\left(\frac{\ell}{r}\right)^{d-3}+O\left(1 / r^{d-2}\right), \quad b(r)=\frac{r^{2}}{\ell^{2}}+\sum_{j=0}^{(d-4) / 2} a_{j}\left(\frac{\ell}{r}\right)^{2 j}+c_{t}\left(\frac{\ell}{r}\right)^{d-3}+O\left(1 / r^{d-2}\right), \\
f(r)=\frac{r^{2}}{\ell^{2}}+\sum_{j=0}^{(d-4) / 2} f_{j}\left(\frac{\ell}{r}\right)^{2 j}+\left(c_{z}+c_{t}\right)\left(\frac{\ell}{r}\right)^{d-3}+O\left(1 / r^{d-2}\right),
\end{gathered}
$$

where $a_{j}, f_{j}$ are constants depending on the index $\kappa$ and of the spacetime dimension only. Their expression can be found in [11. The corresponding expansion for odd values of the spacetime dimension contains $\log$ terms and is given by:

$$
a(r)=\frac{r^{2}}{\ell^{2}}+\sum_{j=0}^{(d-5) / 2} a_{j}\left(\frac{\ell}{r}\right)^{2 j}+\zeta \log \left(\frac{r}{\ell}\right)\left(\frac{\ell}{r}\right)^{d-3}+c_{z}\left(\frac{\ell}{r}\right)^{d-3}+O\left(\frac{\log r}{r^{d-1}}\right),
$$




$$
\begin{aligned}
& b(r)=\frac{r^{2}}{\ell^{2}}+\sum_{j=0}^{(d-5) / 2} a_{j}\left(\frac{\ell}{r}\right)^{2 j}+\zeta \log \left(\frac{r}{\ell}\right)\left(\frac{\ell}{r}\right)^{d-3}+c_{t}\left(\frac{\ell}{r}\right)^{d-3}+O\left(\frac{\log r}{r^{d-1}}\right), \\
& f(r)=\frac{r^{2}}{\ell^{2}}+\sum_{j=0}^{(d-5) / 2} f_{j}\left(\frac{\ell}{r}\right)^{2 j}+2 \zeta \log \left(\frac{r}{\ell}\right)\left(\frac{\ell}{r}\right)^{d-3}+\left(c_{z}+c_{t}+c_{0}\right)\left(\frac{\ell}{r}\right)^{d-3}+O\left(\frac{\log r}{r^{d-1}}\right),
\end{aligned}
$$

where $c_{0}, \zeta$ are constants (supplementing the $a_{j}, f_{j}$ mentionned above) depending on $\kappa, d$ [1], .

The mass of the UBS solutions as evaluated in [11] by using the standard counterterm method is (in this paper we take $G_{d}=1$ )

$$
M=\frac{\ell^{d-4}}{16 \pi}\left[c_{z}-(d-2) c_{t}\right] L V_{\kappa, d-3}+M_{c}^{(\kappa, d)}
$$

where $V_{\kappa, d-3}$ is the total area of the angular sector. $M_{c}^{(\kappa, d)}$ is a Casimir-like term which appears for an odd spacetime dimension only, $M_{c}^{(\kappa, d)}=\frac{\ell^{d-4}}{16 \pi} V_{\kappa, d-3} L\left(\frac{1}{12} \delta_{d, 5}-\frac{333}{3200} \delta_{d, 7}+\ldots\right)$. The Hawking temperature and the entropy of the UBS are given by

$$
T_{H}=\frac{1}{4 \pi} \sqrt{\frac{b_{1}}{r_{h} \ell^{2}}\left[(d-1) r_{h}^{2}+\kappa(d-4) \ell^{2}\right]}, \quad S=\frac{1}{4} r_{h}^{d-3} V_{\kappa, d-3} L \sqrt{a_{h}} .
$$

The thermodynamics of the vacuum AdS UBSs has been discussed to some extend in [11, where it was found that they follow the pattern of the corresponding Schwarzschild-AdS $S_{d}\left(\mathrm{SAdS}_{d}\right)$ black hole solutions. In the more interesting $\kappa=1$ case, the temperature of the black string solutions is bounded from below. At higher temperatures, above a critical value, there exist two bulk solutions that correspond to the so-called small (unstable) and large (stable) black string solutions. Concerning local thermodynamic stability, in the case of a canonical ensemble with a fixed value of the length of the extra-dimension $L$, the response function whose sign determines the thermodynamic stability is the heat capacity $C=T_{H}\left(\partial S / \partial T_{H}\right)_{L}$. The results in [11] indicate that the small UBSs with $\kappa=1$ have negative specific heat (they are thermodynamically unstable) but large size black strings have positive specific heat (and they are stable). The topological black strings $(\kappa=0,-1)$ are thermodynamically stable.

We close this brief review of the UBS properties, by noting that the Einstein equations are left invariant by the transformation $r \rightarrow \bar{r}=\xi r, \quad \ell \rightarrow \bar{\ell}=\xi \ell$. Therefore, one may generate in this way a family of new vacuum solutions, which are usually termed "copies of solutions" [19. The new solutions have the same length in the extra-dimension, the physical quantities vary according to

$$
\bar{r}_{h}=\xi r_{h}, \bar{\Lambda}=\Lambda / \xi^{2}, \quad \bar{T}_{H}=T_{H} / \xi, \quad \bar{M}=\xi^{d-4} M, \quad \bar{S}=\xi^{d-3} S .
$$

As a consequence, given the full spectrum of solutions for a given value of $\Lambda$ (with $r_{\min }<r_{h}<\infty$ ), one may find the corresponding branches for any value of $\Lambda<0$. Equivalently, one can cover the full UBS spectrum by keeping fixed the event horizon radius and varying the value of the cosmological constant $0<|\Lambda|<\infty$.

The problem is thus characterized by two dimensionless parameters $\mu_{1}=M / L^{d-3}, \quad \mu_{2}=L / \ell$. The limit $\mu_{2} \rightarrow 0$ corresponds to black string solutions in a Kaluza-Klein theory.

\section{The Gregory-Laflamme instablity}

The GL mode of the AdS black strings is found in this paper by using the same approach as in 2]. A convenient ansatz for the static NUBS solutions of the Einstein equations with negative cosmological constant is

$$
d s^{2}=-b(r) e^{2 A(r, z)} d t^{2}+e^{2 B(r, z)}\left(\frac{d r^{2}}{f(r)}+a(r) d z^{2}\right)+r^{2} e^{2 C(r, z)} d \Sigma_{\kappa}^{2} .
$$


The UBS limit discussed above corresponds to $A=B=C=0$. This metric form generalizes for the AdS case the usual $\Lambda=0$ NUBS ansatz used e.g. in [3], [5], 6], which is recovered for $\kappa=1$ and $b=1 / f=$ $1-\left(r_{0} / r\right)^{d-4}, a=1$.

Following the standard approach, we perform an expansion around the UBS of the form

$$
A(z, r)=\lambda A_{1}(r) \cos (k z)+O\left(\lambda^{2}\right), \quad B(z, r)=\lambda B_{1}(r) \cos (k z)+O\left(\lambda^{2}\right), \quad C(z, r)=\lambda C_{1}(r) \cos (k z)+O\left(\lambda^{2}\right),
$$

with $\lambda$ a small parameter. The ordinary differential equations for $A_{1}, B_{1}, C_{1}$ are found by plugging (10) into the Einstein equations and retaining the first order in $\lambda$. Following [2], we express the function $B_{1}$ in terms of $A_{1}, C_{1}$ and their first derivatives. This leads to a system of two differential equations:

$$
A_{1}^{\prime \prime}=\alpha_{1} A_{1}+\alpha_{2} A_{1}^{\prime}+\alpha_{3} C_{1}+\alpha_{4} C_{1}^{\prime}, \quad C_{1}^{\prime \prime}=\varphi_{1} A_{1}+\varphi_{2} A_{1}^{\prime}+\varphi_{3} C_{1}+\varphi_{4} C_{1}^{\prime},
$$

where

$$
\begin{aligned}
& \alpha_{1}=\frac{2 b\left((-3+d) k^{2} l^{2}+(r-d r) a^{\prime}\right)+r\left(k^{2} \ell^{2}+2(-1+d) a\right) b^{\prime}}{\ell^{2} a f\left(2(-3+d) b+r b^{\prime}\right)}, \\
& \alpha_{2}=-\frac{1}{r}-\frac{b^{\prime}}{2 b}-\frac{1}{\ell^{2} r f}\left[(-1+d) r^{2}+(-4+d) \ell^{2} \kappa-\frac{4(-1+d) r^{2} b}{2(-3+d) b+r b^{\prime}}\right], \\
& \alpha_{3}=\frac{2(-3+d)(-1+d) b\left(2 a-r a^{\prime}\right)}{\ell^{2} a f\left(2(-3+d) b+r b^{\prime}\right)}, \quad \alpha_{4}=-\frac{(-3+d) b^{\prime}}{2 b}+\frac{4(-3+d)(-1+d) r b}{\ell^{2} f\left(2(-3+d) b+r b^{\prime}\right)}, \\
& \varphi_{1}=\frac{2\left((-1+d) r^{2}+(-4+d) \ell^{2} \kappa\right)\left(-b a^{\prime}+a b^{\prime}\right)}{\ell^{2} r a f\left(2(-3+d) b+r b^{\prime}\right)}, \quad \varphi_{2}=\frac{1}{r}\left[-1+\frac{4 b\left((-1+d) r^{2}+(-4+d) \ell^{2} \kappa\right)}{\ell^{2} f\left(2(-3+d) b+r b^{\prime}\right)}\right], \\
& \varphi_{3}=\frac{2 b(-3+d)\left(k^{2} \ell^{2} r+2(-1+d) r a-\left((-1+d) r^{2}+(-4+d) \ell^{2} \kappa\right) a^{\prime}\right)+\ell^{2}\left(k^{2} r^{2}-2(-4+d) \kappa a\right) b^{\prime}}{\ell^{2} r a f\left(2(-3+d) b+r b^{\prime}\right)}, \\
& \varphi_{4}=\frac{2 b(-3+d)\left(k^{2} \ell^{2} r+2(-1+d) r a-\left((-1+d) r^{2}+(-4+d) \ell^{2} \kappa\right) a^{\prime}\right)+\ell^{2}\left(k^{2} r^{2}-2(-4+d) \kappa a\right) b^{\prime}}{\ell^{2} r a f\left(2(-3+d) b+r b^{\prime}\right)} .
\end{aligned}
$$

This eigenvalue problem for the wavenumber $k=2 \pi / L$ was solved numerically with suitable boundary conditions. The solutions of (12) were constructed in a systematic way for $5 \leq d \leq 8$ and $\kappa=0, \pm 1$; a number of solutions with $d=9,10,11$ have been also considered. To integrate the equations, we used the differential equation solver COLSYS which involves a Newton-Raphson method [20. At $r=r_{h}$ the regularity of the solution imposes specific relations between $A_{1}, C_{1}$ and their first derivatives; these relations are very long and we prefer not to include them here. Also, the perturbation has to vanish at $r \rightarrow \infty$, i.e. $\lim _{r \rightarrow \infty} A_{1}, C_{1}=0$.

Given the linearity of the equations, there is also a freedom in the choice of the normalisation of the solutions. Solutions obeying the appropriate boundary conditions exist only for specific values of the spectral parameter $k^{2}$. In practice, we first computed the background functions $a, b$ and $f$, and then solve the equations (11) supplemented by the equation $d k^{2} / d r=0$. The supplementary boundary condition allowed by this extra equation can then be imposed in order to eliminate the arbitrariness of the normalisation (we choose $C_{1}\left(r_{h}\right)=1$ ) and leads to a well posed problem. Once given a starting profile sufficiently close to the solution, the numerical solver is able to construct with a very good accuracy both the perturbation and the corresponding value of $k^{2}$ for a given set of the parameters $\left(\kappa, d, r_{h}, l^{2}\right)$. A positive value for $k^{2}$ indicates the presence of an unstable mode of the background UBS configuration.

Starting with the more familiar solutions with an event horizon topology $S^{d-3} \times S^{1}$, we noticed the existence, for a fixed value of $r_{h}$, of a critical value of the cosmological constant $\Lambda_{c}$, beyond which the AdS black strings become stable. The value of $\Lambda_{c}$ is dimension dependent; one finds e.g. $\Lambda_{c}(d=5) \simeq-1.27$, $\Lambda_{c}(d=6) \simeq-3.52$ (the event horizon radius here is $\left.r_{h}=1\right)$. However, all solutions with $\Lambda$ between zero and $\Lambda_{c}$ are classical unstable. The precise value of the wave-number $k$ depends on $d$ and $\Lambda$.

Taking instead a fixed value of the cosmological constant and varying the event horizon radius, this translates into the existence of a critical $r_{h}^{c}$ above which the solutions are stable (note that the product $k \ell$ is invariant under the rescaling of the radial coordinate). These results are presented in Figure 1. One can also see that the quantity $k^{2}$ is positive and diverges as $r_{h} \rightarrow 0$. This demonstrates that AdS globally regular 


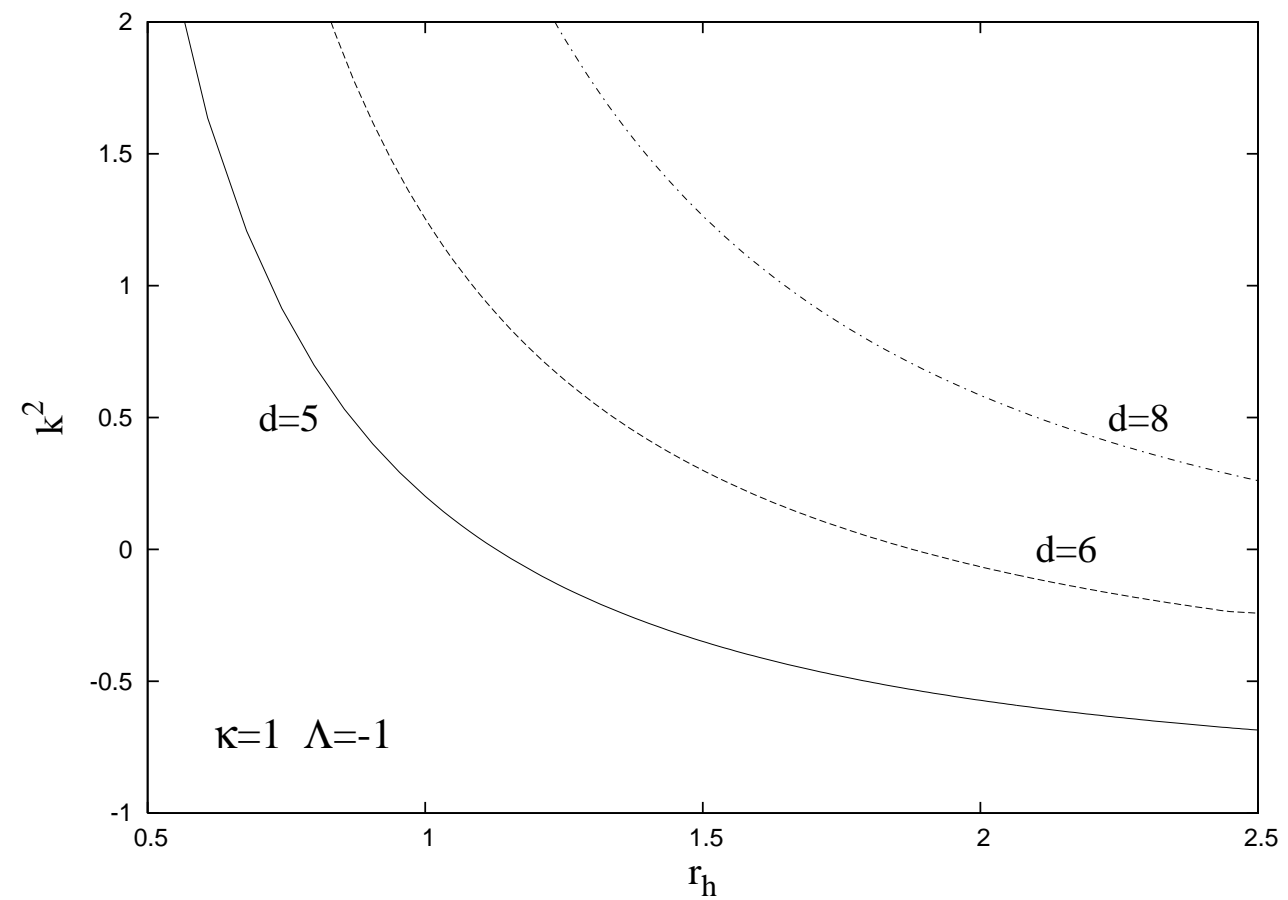

Figure 1. The square of the critical wave-number $k^{2}$ is plotted as a function of the horizon radius $r_{h}$ for black string solutions with an event horizon topology $S^{d-3} \times S^{1}$ and several spacetime dimensions.

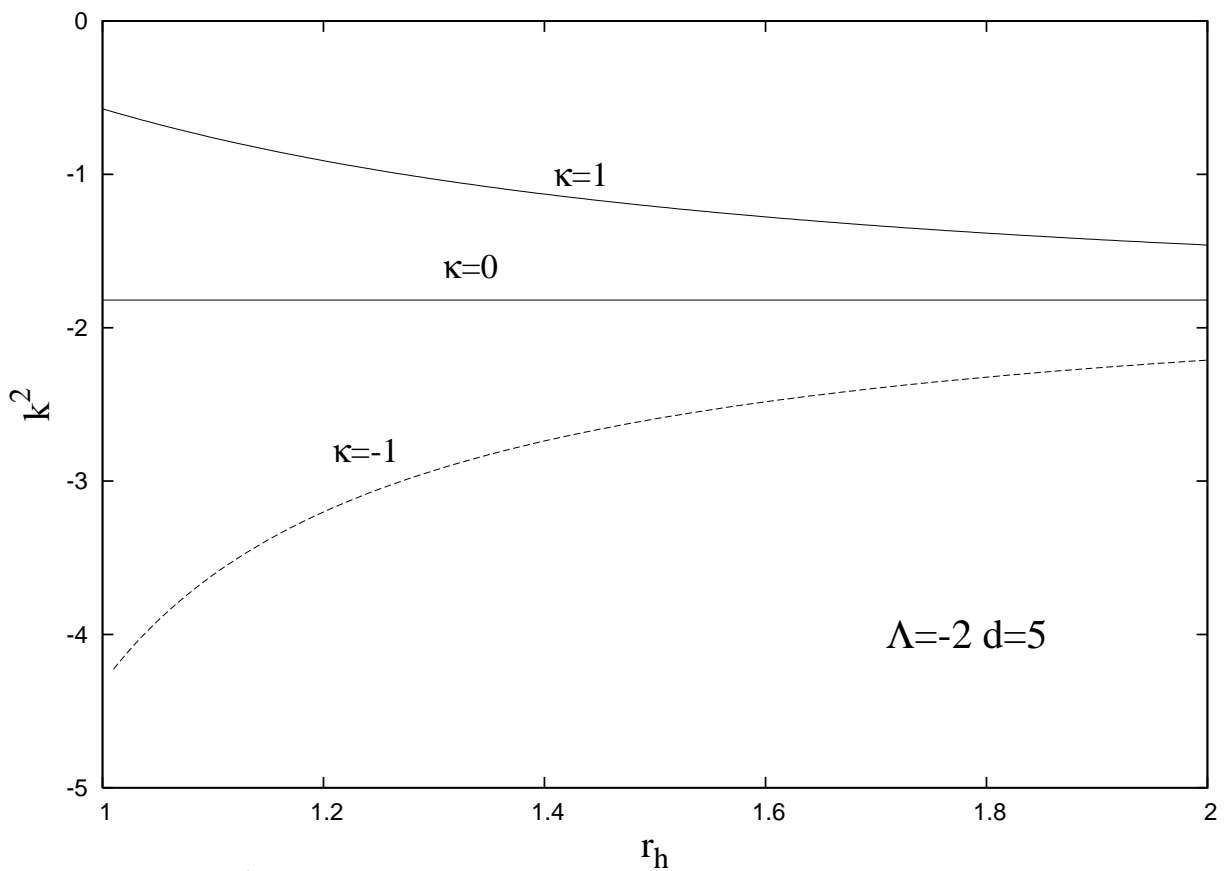

Figure 2. The same as Figure 1 for $\Lambda=-2, d=5$ black string solutions with $\kappa=-1,0,1$.

UBS solution is classically unstable.

The picture for topological black strings $(\kappa=0,-1)$ is very different. Our findings indicate that the topological black string solutions do not present an instability within the ansatz (9), (10). The numerics indeed gives $k^{2}<0$ for all values of $\left(r_{h}, \Lambda\right)$ that we have considered. The dependance of the eigenvalue $k^{2}$ on the horizon radius $r_{h}$ for the three possible topologies (in the allowed region for $\kappa=-1$ ) is reported in Figure 2 for $d=5$ black strings with $\Lambda=-2$. The value corresponding to $\kappa=0$ is $r_{h}$-independent and turns out to constitute a lower (resp. upper) bound for the case $\kappa=1$ (resp. $\kappa=-1$ ). 

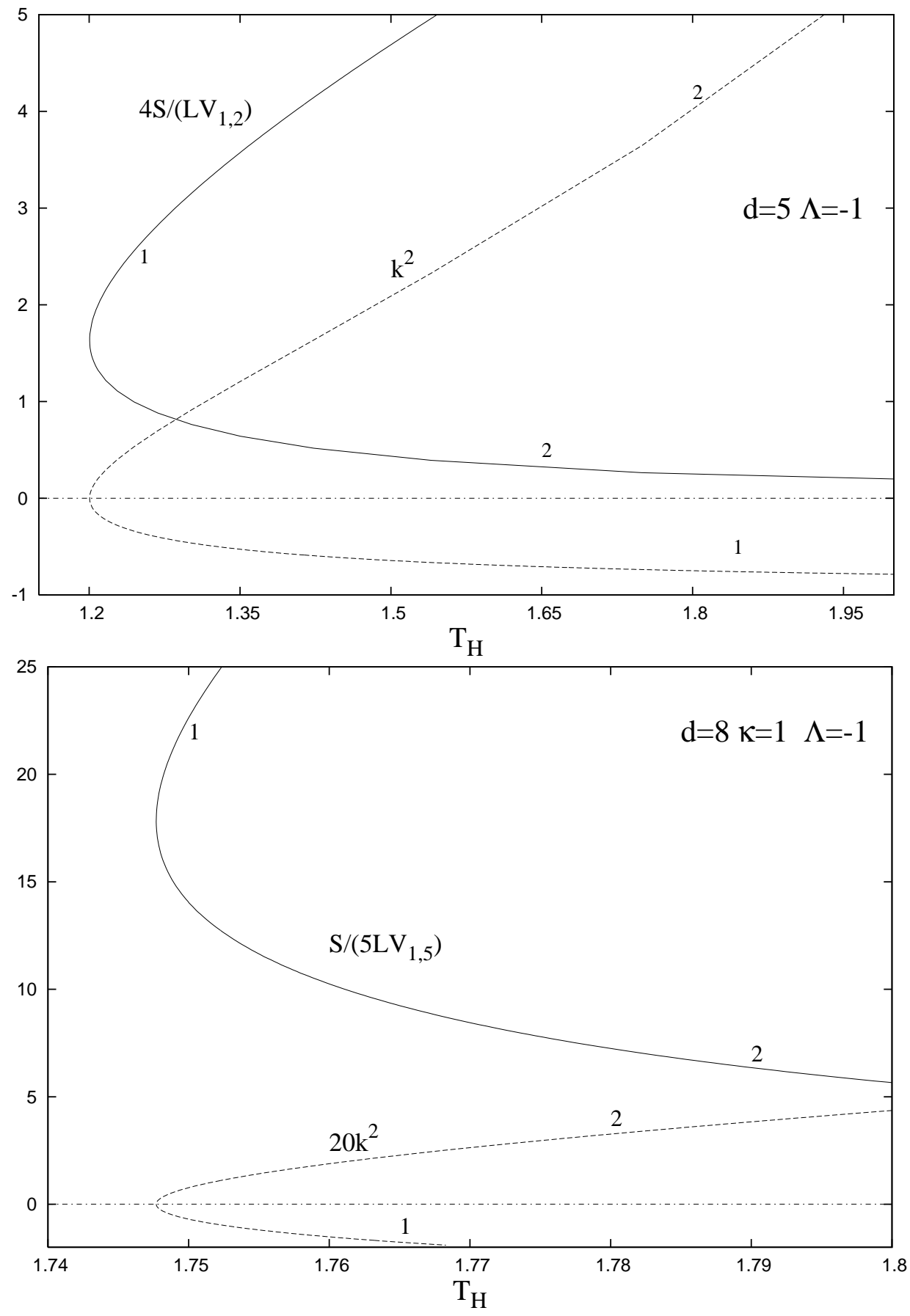

Figure 3. The entropy per unit surface $S /\left(4 L V_{\kappa, d-3}\right)$ and the square of the critical wave-number $k^{2}$ are plotted as a function of the Hawking temperature $T_{H}$ for black string solutions with an event horizon topology $S^{d-3} \times S^{1}$ in $d=5$ and $d=8$ spacetime dimensions (for a better visualisation, both $S$ and $k^{2}$ are multiplied with suitable factors).

It is also interesting to notice that $k^{2}$ is well fitted by a function of the form

$$
k^{2} \simeq \alpha+\frac{\beta}{r_{h}^{c}}
$$

the best fit for the parameters $a, b, c$ gives $\alpha \simeq-1.85, \beta \approx 1.28, c \simeq 1.68$ for $\kappa=1$ and $\alpha \simeq-2.02, \beta \simeq$ $-2.22, c \simeq 3.4$ for $\kappa=-1$ and $\alpha=-1.82, \beta=0$ for $\kappa=0$ (with $\Lambda=-2$ ). 


\section{Further remarks}

On general grounds, one expects at least the small AdS black strings to present a GL-type instability. This was confirmed by our numerical results discussed in the previous section for black strings with an horizon topology $S^{d-3} \times S^{1}$. Moreover, we have found that all topological black strings are stable with respect to perturbations of the form (10) emphasized in this paper.

We expect the results of this paper to be relevant for the issue of AdS black rings (yet to be found). As observed in [11, the heuristic construction of black rings (see e.g. 21]) applies also for $\Lambda<0$, and one expects an AdS black ring to approach the UBS black string solution in the limit where the radius of the ring circle grows very large. Thus, in a similar way to the asymptotically flat case [22, some of the AdS black ring solutions will also present a GL-type instability.

The results in the previous Section provide also an explicit realization of the GM conjecture. This is illustrated in Figure 3, where we plot the wavelength $k$ and the entropy $S$ as a function of temperature $T_{H}$ for $\kappa=1$ black strings with a fixed value of $\Lambda$, in $d=5,8$ spacetime dimensions. One can see in particular that the solutions with $k^{2}<0$ have also a positive specific heat (this branch has the index 1 in that plot), while the entropy decreases with $T_{H}$ along the branch with $k^{2}>0$ (index 2 in the Figure 3 ). In agreement with the GM conjecture, the critical temperature where $k^{2}$ crosses zero coincides (within the numerical accuracy) with the temperature at the turning point of the UBS branches in a $\left(S-T_{H}\right)$ diagram $\left(e . g . T_{H}^{c} \simeq 1.2005\right.$ for $d=5$ and $T_{H}^{c} \simeq 1.7477$ for $d=8$ ). A similar picture has been found for other spacetime dimensions. When considering instead topological black strings, the result $k^{2}<0$ is, indeed, what we expect since these solutions have always a positive specific heat [11].

In principle, following [1, one can get an estimation of the critical wave-number $k$ by equating the entropy of the UBS with that of SAdS black hole in $d$-dimensions with the same mass and the same topological parameter $\kappa$. The relation between the entropy and the mass of a $\mathrm{SAdS}_{d}$ black hole is

$$
\kappa-\frac{M}{\frac{(d-2) V_{\kappa, d-2}}{16 \pi}} \frac{1}{\left(4 S / V_{\kappa, d-2}\right)^{(d-3) /(d-2)}}+\left(\frac{4 S}{V_{\kappa, d-2}}\right)^{\frac{2}{d-2}} \frac{1}{\ell^{2}}=0,
$$

(here we ignore the Casimir-like mass terms which appear for an odd $d$ ). For a UBS solution with the same values of mass and entropy, one can use (6), (7) to write $S=V_{\kappa, d-3} L \ell^{d-3} n_{1} / 4, M=V_{\kappa, d-3} L \ell^{d-4} n_{2} /(16 \pi)$, with the numerical coefficients $n_{1}=\sqrt{a_{h}}\left(r_{h} / \ell\right)^{d-3}, \quad n_{2}=c_{z}-(d-2) c_{t}$. This yields the following equation for the critical ratio $y=\mu_{2}^{1 /(d-2}=(L / \ell)^{1 /(d-2)}$,

$$
c_{1} y^{2}+c_{2} y+k=0,
$$

where $c_{1}=n_{1}^{2 /(d-2)}\left(V_{\kappa, d-3} / V_{\kappa, d-2}\right)^{2 /(d-2)}, c_{2}=-\left(V_{\kappa, d-3} / V_{\kappa, d-2}\right)^{1 /(d-2)}\left(n_{2} / n_{1}^{(d-3) /(d-2)}\right) \frac{1}{d-2}$. For $\kappa=1$, this argument predicts the existence of a critical value of $r_{h}$ above which the black strings are stable (i.e. no real solutions of the equation (15)). However, it also gives two possible values for the ratio $L / \ell$ (which are in the same range with what we have found numerically). Moreover, this argument predicts an instability of the topological black strings for suitable values of the event horizon area, which appear not to occur. This may be due to the fact that the SAdS black holes would fail to provide a suitable lowest order approximation for the hypothetical AdS counterparts of the $\Lambda=0$ caged black holes.

Concerning more general solutions, the study of the perturbative equations in second order for static $\Lambda=0$ black strings, revealed the appearance of a critical dimension, above which the perturbative nonuniform black strings are less massive than the marginally stable uniform black string [4. It would therefore be interesting to solve the perturbative equations to higher order also for AdS solutions. Also, the unstable black strings are part of a larger phase diagram. Other classes of AdS black objects, presenting the same structure of the conformal infinity but a different topology of the event horizon are likely to exist.

\section{Acknowledgments}

YB gratefully acknowledges the Belgian FNRS for financial support. The work of ER was supported by a fellowship from the Alexander von Humboldt Foundation 


\section{References}

[1] R. Gregory and R. Laflamme, Phys. Rev. Lett. 70 (1993) 2837 arXiv:hep-th/9301052.

[2] S. S. Gubser, Class. Quant. Grav. 19 (2002) 4825 arXiv:hep-th/0110193.

[3] T. Wiseman, Class. Quant. Grav. 20 (2003) 1137 arXiv:hep-th/0209051.

[4] E. Sorkin, Phys. Rev. Lett. 93 (2004) 031601 arXiv:hep-th/0402216.

[5] B. Kleihaus, J. Kunz and E. Radu, JHEP 0606 (2006) 016 arXiv:hep-th/0603119.

[6] E. Sorkin, Phys. Rev. D 74 (2006) 104027 arXiv:gr-qc/0608115.

[7] B. Kol, Phys. Rept. 422 (2006) 119 arXiv:hep-th/0411240.

[8] T. Harmark, V. Niarchos and N. A. Obers, Class. Quant. Grav. 24 (2007) R1 arXiv:hep-th/0701022.

[9] K. Copsey and G. T. Horowitz, JHEP 0606 (2006) 021 arXiv:hep-th/0602003.

[10] A. Chamblin, S. W. Hawking and H. S. Reall, Phys. Rev. D 61 (2000) 065007 arXiv:hep-th/9909205.

[11] R. B. Mann, E. Radu and C. Stelea, JHEP 0609 (2006) 073 arXiv:hep-th/0604205.

[12] Y. Brihaye, E. Radu and C. Stelea, arXiv:hep-th/0703046.

[13] Y. Brihaye and E. Radu, arXiv:0706.4378 [hep-th].

[14] A. Bernamonti, M. M. Caldarelli, D. Klemm, R. Olea, C. Sieg and E. Zorzan, arXiv:0708.2402 [hep-th];

A. H. Chamseddine and W. A. Sabra, Phys. Lett. B 477, 329 (2000) arXiv:hep-th/9911195;

D. Klemm and W. A. Sabra, Phys. Rev. D 62, 024003 (2000) [arXiv:hep-th/0001131];

W. A. Sabra, Phys. Lett. B 545, 175 (2002) arXiv:hep-th/0207128].

[15] Y. Brihaye and T. Delsate, Phys. Rev. D 75 (2007) 044013 arXiv:hep-th/0611195.

[16] S. S. Gubser and I. Mitra, arXiv:hep-th/0009126;

S. S. Gubser and I. Mitra, JHEP 0108 (2001) 018 arXiv:hep-th/0011127.

[17] T. Hirayama and G. Kang, Phys. Rev. D 64 (2001) 064010 arXiv:hep-th/0104213;

U. Miyamoto, arXiv:0709.1028 [hep-th].

[18] J. J. Friess, S. S. Gubser and I. Mitra, Phys. Rev. D 72 (2005) 104019 arXiv:hep-th/0508220.

[19] T. Harmark and N. A. Obers, Nucl. Phys. B 684 (2004) 183 arXiv:hep-th/0309230.

[20] U. Ascher, J. Christiansen, R. D. Russell, Math. of Comp. 33 (1979) 659;

U. Ascher, J. Christiansen, R. D. Russell, ACM Trans. 7 (1981) 209.

[21] R. Emparan, T. Harmark, V. Niarchos, N. A. Obers and M. J. Rodriguez, arXiv:0708.2181 [hep-th].

[22] H. Elvang, R. Emparan and A. Virmani, JHEP 0612 (2006) 074 arXiv:hep-th/0608076. 